\title{
Spectrophotometric intracutaneous analysis: a new technique for imaging pigmented skin lesions
}

\author{
M.MONCRIEFF, ${ }^{*} \dagger$ S.COTTON, + E.CLARIDGE $§$ AND P.HALL* \\ *Department of Plastic and Reconstructive Surgery, Addenbrooke's Hospital, Box 186, Hills Road, Cambridge CB2 2QQ, U.K. \\ $\dagger$ †Department of Plastic and Reconstructive Surgery, West Norwich Hospital, Norwich NR2 3TU, U.K. \\ $\ddagger$ Astron Clinica, Cambridge, U.K. \\ §School of Computer Science, University of Birmingham, Birmingham, U.K.
}

Accepted for publication 12 September 2001

Summary Background Spectrophotometric intracutaneous analysis (SIA) is a new technique for imaging pigmented skin lesions and for diagnosing melanoma. The SIAscope produces eight narrow-band spectrally filtered images of the skin over an area of $24 \times 24 \mathrm{~mm}$ with radiation ranging from 400 to $1000 \mathrm{~nm}$.

Objectives To present the early results of a clinical trial with SIA.

Methods Spectrophotometric inputs from the skin were analysed using complex algorithms to return high-resolution information regarding total melanin content of the epidermis and papillary dermis, collagen and haemoglobin content as well as the presence of melanin in the papillary dermis.

Results Simple, highly reproducible and reliable features were identified, e.g. the presence of dermal melanin, collagen holes and 'erythematous blush' with blood displacement. These simple features were found to be highly specific $(80 \cdot 1 \%)$ and sensitive $(82 \cdot 7 \%)$ for melanoma in a dataset of 348 pigmented lesions ( 52 melanomas) and compared very favourably with dermatoscopy when analysed using receiver-operator characteristic curves.

Conclusions This first clinical trial with SIAscopy has yielded very promising results and delivers new, useful information to the clinician diagnosing pigmented skin lesions.

Key words: dermatoscopy, diagnosis, imaging, melanoma, SIAscopy, spectrophotometric intracutaneous analysis

The overwhelming majority of the literature documents the relentless world-wide progress of melanoma up the incidence league tables of all cancers in both incidence and mortality. The highest incidence is to be found in the Antipodes where a recent report from New Zealand $^{1}$ indicates that Auckland has overtaken Queensland, Australia ${ }^{2}$ with a staggering 78 per 100, 000 (crude rate). By comparison, the overall incidence of melanoma in the U.K. in 1995 was reported at 5000 cases per annum (written communication from Department of Medical Statistics, Cancer Research Campaign, London). Similarly, mortality statistics throughout the world show a changing trend. Crude mortality measurements show that this is still

Correspondence: Marc Moncrieff.

E-mail: marc.moncrieff@cwcom.net rising, ${ }^{3}$ although the rate of increase is trailing off. Statistics such as these have led to the dramatic rise being termed as an 'epidemic' and 'an emerging medical catastrophe'. ${ }^{4}$ Of all the prognostic factors, both clinical and histopathological, Breslow thickness of the primary lesion is by far the most important. ${ }^{5}$ Prognosis is so intrinsically linked with this measurement that apparent differences in survival between patient groups are often negated or greatly reduced when they are stratified for tumour thickness. In addition, vertical growth phase melanomas have a worse prognosis that is dependent on other factors. ${ }^{6}$ In essence, early diagnosis and excision are the key to the likely survival of the patient.

The clinical diagnosis of melanoma is acknowledged as challenging by most authors and has provoked much debate. ${ }^{7-9}$ Diagnostic accuracy has been stated

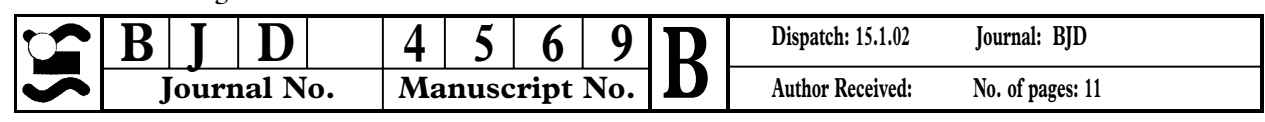


as being between $50 \%$ and $80 \%$. Grin et al. ${ }^{8}$ demonstrated a small improvement in accuracy with time (60\% vs. $64 \%$ ), and Morton and MacKie ${ }^{9}$ demonstrated a significant improvement in accuracy with an examiner of 10 years' experience or more $(60 \%$ vs. $80 \%)$. As a result of the difficult task that clinicians can face in achieving an early diagnosis of a heterogeneous disease entity, there arises the need for tools that can improve diagnostic accuracy. Systems that have been developed as adjuncts in the diagnosis of melanoma include highfrequency ultrasound, optical coherence tomography, confocal microscopy and magnetic resonance imaging. ${ }^{10-13}$ Some or all of the following limit these techniques: availability, expense, imaging resolution and lack of useful diagnostic detail. However, dermatoscopy (epiluminescence microscopy, dermoscopy, oilemersion microscopy, skin-surface microscopy) is a useful technique that uses oil and a hand-held microscope, which allows visualization of morphological characteristics at the dermoepidermal junction. Clinicians then attempt to diagnose the presence of melanoma by analysing the lesion by colour, pattern and specific morphological features. In 1990, a consensus paper was published to standardize the terminology used to describe features seen in the surface microscopy of pigmented skin lesions and correlated them with their underlying histology. ${ }^{14}$ Since then, a whole wealth of literature, papers and textbooks has been published regarding this technique. A critical and systematic review of this literature ${ }^{15}$ found that where the specificity and sensitivity of the clinician was high for clinical diagnosis then the dermatoscope had little to offer in addition. ${ }^{16,17}$ However, using the dermatoscope with equivocal lesions increased the sensitivity for diagnosis between $10 \%$ and $27 \%{ }^{18,19}$

The identification of dermatoscopy features is based on a visual interpretation of colours within a dermatoscopic view. These colours depend upon reflection and absorption by chromophores within the superficial skin according to their depth and concentration and also upon the wavelength of incident light interacting with them. However, we have shown ${ }^{20-22}$ that it is possible for the same colour to arise from different combinations of microscopic structures. This complication may explain the high subjectivity in identifying dermatoscopic features, with the possibility of diagnostic error as a result. As an example, bluegrey areas are defined as 'superficial fibrosis with melanophages and/or malignant cells in the papillary dermis'. ${ }^{14}$ This feature has a specificity of $97 \%$ for invasive melanoma and has been described as the single most important surface microscopic finding' in making a diagnosis. ${ }^{23}$ However, the same appearance can also be produced by a reduction in the thickness of the papillary dermis or an overlying layer of hyperkeratosis. ${ }^{20}$ Another complicating factor of dermatoscopy is that the visual appearance of a skin lesion through a dermatoscope is the result of the layering of microscopic structures. As an analogy, it is akin to looking down on the surface of a rain forest from a helicopter and trying to identify a particular type of fern through the layers of different trees and bushes. It is clear that dermatoscopy can perform a valuable role in the investigation of pigmented skin lesions by increasing the information available for consideration in what is often a difficult diagnostic task. This extra information is particularly useful because it allows, by visual interpretation of the dermatoscopy features, an understanding of the in vivo microarchitecture of the skin lesion. However, it is the necessarily subjective identification of these, often subtle, features that leads dermatoscopy to require thorough training and experience. As a result, dermatoscopy requires a steep learning curve. Studies have shown that trainees may produce a decrease in sensitivity for the diagnosis of melanoma at the outset of learning when compared with performance on clinical grounds alone. ${ }^{19}$

It was to address the operator-dependent and subjective nature of dermatoscopy in extracting information regarding the microstructure of a skin lesion that the SIAscope was developed. Spectrophotometric intracutaneous analysis (SIA) arose from research undertaken at the University of Birmingham in the U.K. where the colours arising from skin lesions were being investigated with the aim of identifying objective diagnostic information. ${ }^{20-22}$ It became apparent that analysis of purely visual colour information was insufficient to extract reliable diagnostic information relating to the lesion's internal structure and that to extract such information it was necessary to extend the analysis into the infrared. The result of this research is a technique capable of rapidly imaging a skin lesion in vivo and allowing examination of the distribution, position and quantity of a range of chromophores, including melanin, blood and collagen within the papillary dermis and the position of melanin relative to the dermoepidermal junction. The clinical device employing this technique is termed a SIAscope and returns SIA graphs showing these constituents for interpretation.

The SIAscope (Fig. 1). operates by probing the skin spectrally over an area of $24 \times 24 \mathrm{~mm}$ or $12 \times 12 \mathrm{~mm}$ 


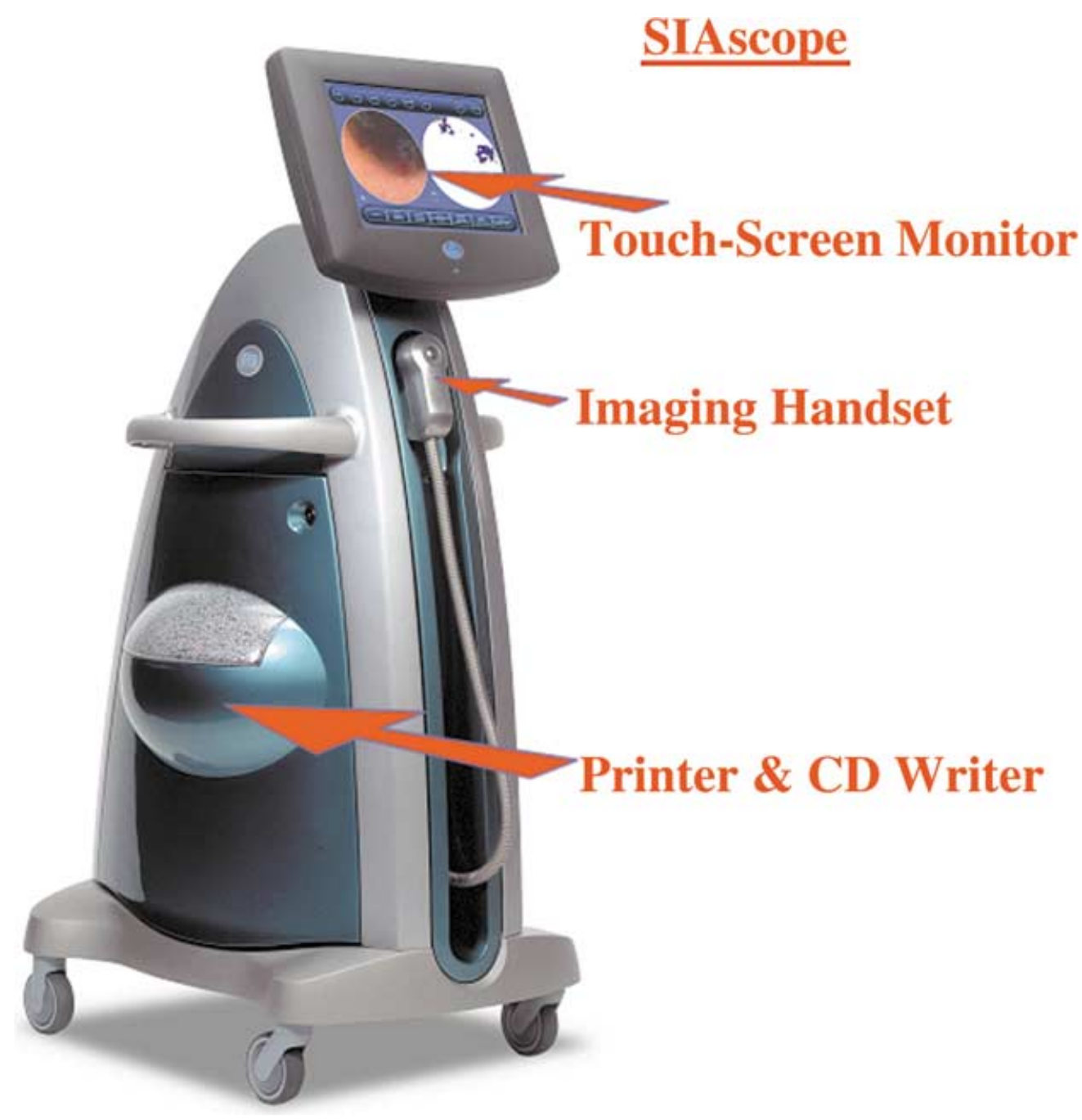

Figure 1. A SIAscope.

with radiation ranging from 400 to $1000 \mathrm{~nm}$. For this trial the larger, 24-mm handset was used. Eight narrow-band spectrally filtered images of the skin were obtained, calibrated and used as inputs to a series of complicated computer algorithms that extract information regarding the microarchitecture of the skin. The wavelengths of the wavebands were chosen to interact preferentially with constituents of the skin. ${ }^{20-22}$ The SIA algorithm first utilizes the infrared wavebands to ascertain the quantity of collagen within the papillary dermis for every point over the skin lesion. This is the crucial step for this technique and provides a necessary transformation on the wavebands allowing accurate extraction of total melanin and blood. The total melanin, collagen and blood SIA graphs can now be displayed. The effect of these chromophores on the wavebands is then removed, allowing a key piece of diagnostic information, the presence of melanin below the dermoepidermal junction, to be identified. This is possible because the spectral remittance of melanin changes with regard to its position in the superficial anatomical layers of the skin, namely the epidermis and the papillary dermis. Using SIAscopy the accuracy of this identification is extremely high and forms the dermal melanin SIA graph. ${ }^{21}$

In this paper, we aim to introduce the technique of SIA and to present the early results of a clinical trial under way in the U.K.

\section{Materials and methods}

The study was designed to enable the identification of features from SIA graphs that may assist in the early identification of melanoma when compared with the 
histopathological examination from excisional biopsy of pigmented skin lesions. Patients referred for excisional biopsy of a pigmented skin lesion at either the plastic surgery or dermatology departments at Addenbrooke's and West Norwich Hospitals were recruited to the study and informed consent was obtained. The patients were photographed twice using conventional 35-mm slide film (Kodachrome ISO 64): once using a standard $\times 2$ macro lens (Canon) and once using a dermatoscopy lens (Heine Dermaphot: $\times 10$ magnification). Patients were asked questions about the lesion-change in size, shape and colour; change in sensation; bleeding and inflammation-and the maximum diameter of the lesion was measured in millimetres. This information and standard demographic data (age, sex, name and hospital number) were recorded and stored in a protected computerized database. SIAscopy was performed using the SIAscope (Astron Clinica, Cambridge, U.K.) and the returned SIA graphs were also stored on a protected computerized database. The system takes approximately $5 \mathrm{~s}$ to acquire the images and a further $10 \mathrm{~s}$ to process the data and return them as visual information on a 15-inch monitor display using dedicated software (Astron Clinica). Excisional biopsy was then performed and the results from histopathological examination (used as the reference or gold standard) were obtained and matched with the data. Two senior clinicians, one of whom specializes in dermatopathology, made the histopathological diagnosis of melanoma. A proforma was used as a guideline for the final report. Information from this report includes diagnosis, microscopic detail and, in the case of melanoma, subtype, Breslow thickness, Clark's level, regression and growth phase.

Working definitions of possible features that could correlate with underlying significant histopathology were devised for the purpose of analysis (Table 1).
Examples of these features can be seen in Figs 2 and 3. A dataset of 348 lesions collected over a period of 12 months (February 2000-February 2001) was analysed for the presence or absence of each of these features independently by two of the authors (S.C., M.M.) using standard SIAscope viewing facilities. Both experimenters were blinded as to the histopathological diagnosis of the lesion. Statistical analysis was performed on the dataset using SigmaStat version 2.03 and SPSS for Windows version 9.0 (SPSS Inc., Chicago, IL, U.S.A.) software on a personal computer to obtain specificity and sensitivity data for each of the features. Logistic regression analysis was performed to produce this information for combinations of the features. Reproducibility and reliability data were also produced in the form of the $\kappa$ statistic for each of the features. This statistic provides a measure of agreement beyond any agreement that may have occurred by chance, and takes a value between 0 (no agreement above that which would have occurred by chance) and 1 (perfect agreement) with an improving scale of agreement represented by intermediate values. ${ }^{24}$

\section{Results}

The dataset comprised 348 lesions (311 patients: 200 female and 111 male), including 52 melanomas (one in 6.70 lesions). Of the melanomas, six were in situ, 11 had a Breslow thickness $<0.76 \mathrm{~mm}$ and in total 28 lesions had a Breslow thickness $\leq 1.0 \mathrm{~mm}$ (range in situ to $6.0 \mathrm{~mm}$ ). The median and mean Breslow thicknesses were $1.0 \mathrm{~mm}$ and $1.52 \mathrm{~mm}$, respectively, and the subtypes included 41 superficial spreading melanomas, nine nodular and two acral lentiginous melanoma. Most of the non-melanoma lesions were benign naevi, but overall the sample

Table 1. SIAscopy features defined

\begin{tabular}{lll}
\hline Feature & \multicolumn{1}{c}{ Definition } & SIA graph view \\
\hline $\begin{array}{l}\text { Melanin globules } \\
\text { Blood globules }\end{array}$ & A circumscribed aggregation or flocculation of melanin within the lesion & Total melanin \\
Blood displacement & A circumscribed aggregation or flocculation of blood within the lesion & Blood \\
Erythematous blush & A peripheral increase in blood within the lesion compared with the surrounding & Blood \\
& normal skin for $3 / 4$ of the circumference of the lesion & Blood \\
Dermal melanin & The presence of dermal melanin within the lesion not due to hairs & Dermal melanin \\
Dermal melanin globules & A circumscribed aggregation or flocculation of dermal melanin within the lesion & Dermal melanin \\
Collagen holes & An area within the lesion coinciding with melanin (dermal or total) demonstrating & Collagen \\
& an absence of collagen not due to hair follicles or sebaceous/sweat ducts & Total melanin \\
Biaxial symmetry & Symmetry of shape and pattern in two perpendicular planes within the & \\
& constraints of biological variability & Total melanin \\
Asymmetry & A lack of symmetry of shape and pattern in any plane &
\end{tabular}




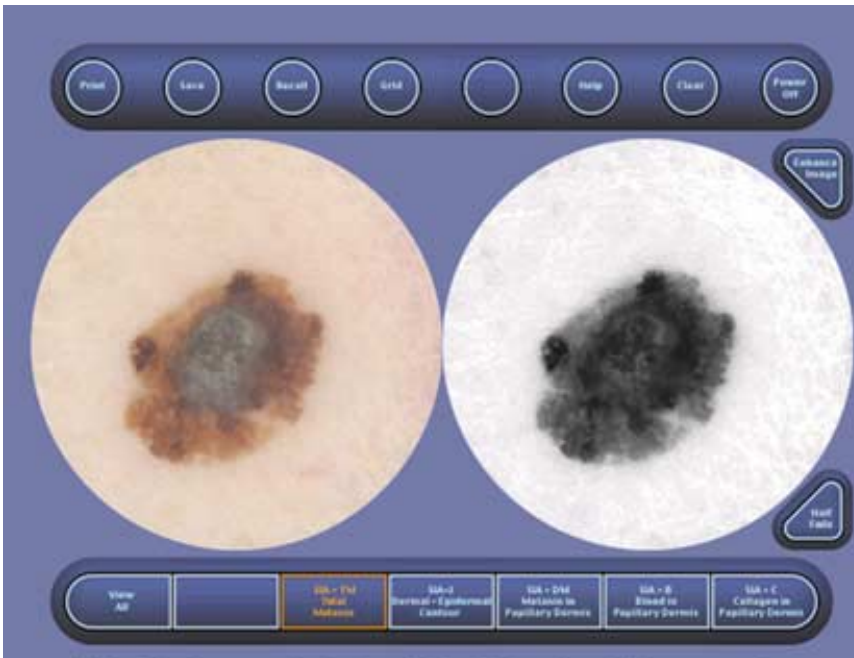

(A) Colour \& Total Melanin Siagraphs

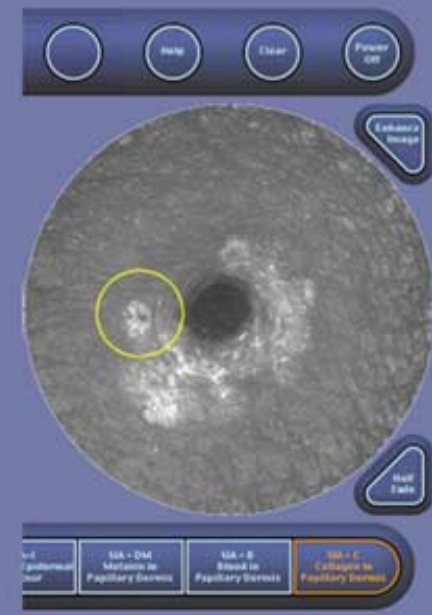

(C) Collagen Siagraph

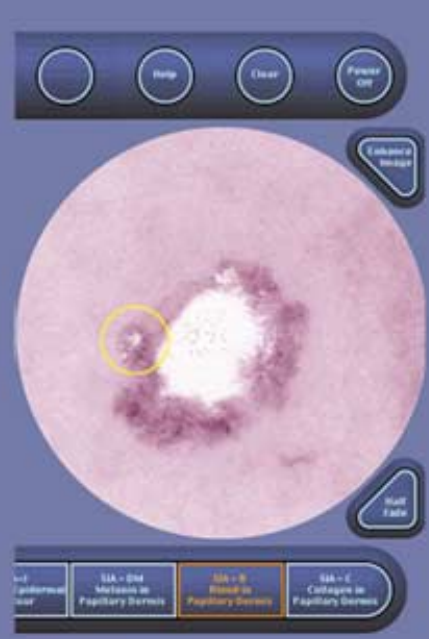

(B) Blood Siagraph

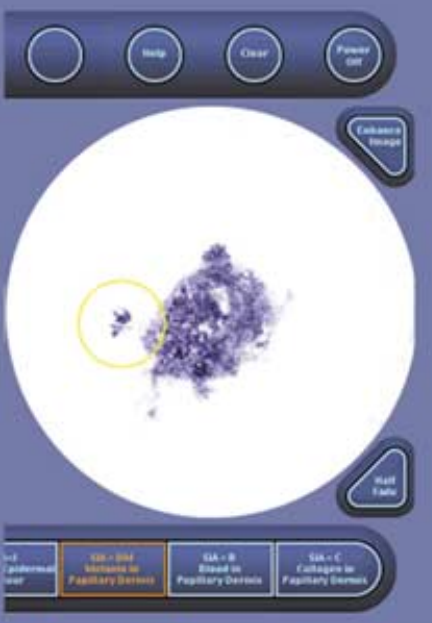

(D) Dermal Melanin Siagraph

Figure 2. SIA graphs of a superficial spreading melanoma (vertical growth phase, $2.5 \mathrm{~mm}$ Breslow thickness, Clark's level IV). (A) Colour SIA graph (left) shows dermatoscopic view, especially blue-grey veil. Total melanin SIA graph (black = melanin) enhances dermatoscopic criteria such as branched streaking and pseudopodia (right). (B) Blood SIA graph (red = blood). The blood displacement is seen as a white area in the vertical growth phase nodule in the centre and also less obviously at the periphery at 9 o'clock (circled). The erythematous blush is quite striking in this lesion. (C) Collagen SIA graph (white = collagen). The collagen hole is very large in the centre of this lesion. There is also a small collagen hole at the periphery at 9 o'clock (circled). (D) Dermal melanin SIA graph (blue = dermal melanin present). There are large quantities of dermal melanin irregularly distributed in this lesion. There is also a separate focus at the periphery at 9 o'clock (circled).

demonstrated a case mix that a clinician would usually be presented with in a dermatology clinic (Table 2).

Each feature was measured for its reliability (interobserver) and reproducibility (intraobserver) by using the $\kappa$ statistic. Most features were highly reproducible and reliable (Figs 4 and 5), indicating good working definitions. ${ }^{24}$ Two features, namely blood globules and melanin globules, were found to be unreliable and irreproducible and so were discarded from further statistical analysis. As expected, no single feature was both highly specific and sensitive: if a feature was highly sensitive, e.g. the presence of dermal melanin, it was at the expense of poor specificity (Table 3). Combinations of features were then analysed using multiple logistic regression analysis. Consistently useful and specific features were the presence of dermal melanin and blood displacement with erythematous 


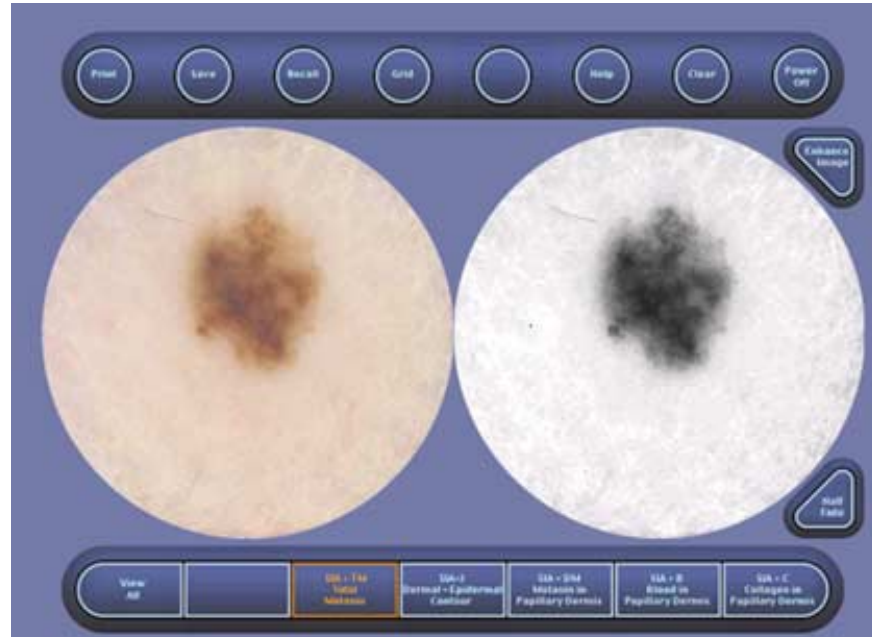

(A) Colour \& Total Melanin Siagraphs

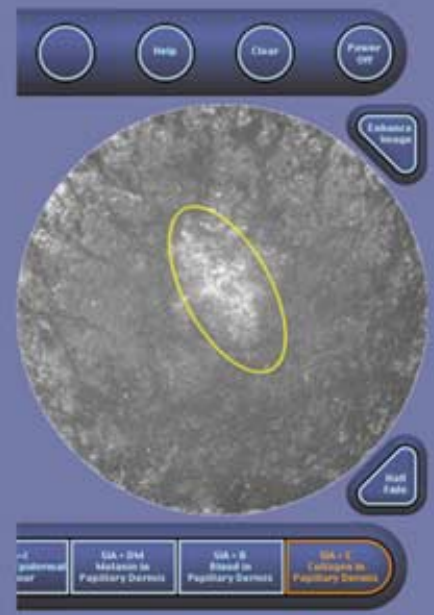

(C)Collagen Siagraph

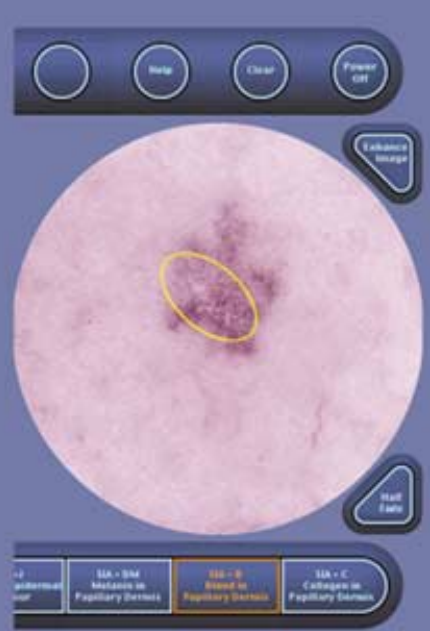

(B) Blood Siagraph

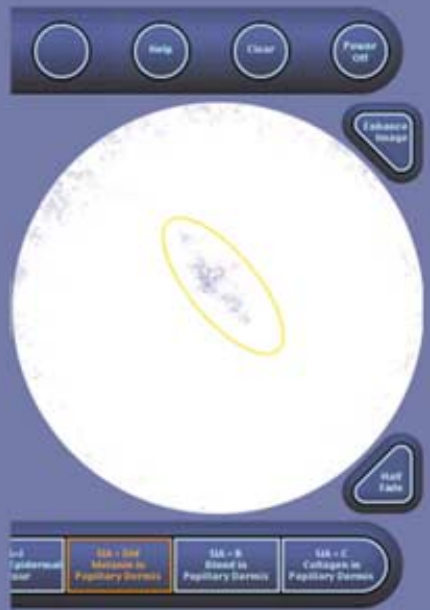

(D) Dermal Melanin Siagraph

Figure 3. SIA graphs of a superficial spreading melanoma (vertical growth phase, $0 \cdot 3 \mathrm{~mm}$ Breslow thickness, Clark's level II). This early lesion demonstrates how SIAscopy may assist in the diagnosis of melanoma. The colour and total melanin SIA graphs (A) are unremarkable. However, the blood SIA graph (B) shows a subtle blood displacement with erythematous blush (circled). The collagen SIA graph (C) shows no holes as this is only a Clark's level II melanoma, although there are large quantities of irregular collagen (circled) consistent with fibrosis. The dermal melanin SIA graph (D) shows dermal melanin irregularly distributed across a large area of the lesion (circled).

blush, and the inclusion of the presence of collagen holes as a further feature increased the sensitivity. It was then decided to investigate the effect of adding clinical information to try and improve the specificity and sensitivity of the test and to approximate the timehonoured clinical progression of history, examination and investigation of the patient. It was found that for lesions of $6 \mathrm{~mm}$ and greater with dermal melanin and blood blush/displacement there was a sensitivity of $82 \cdot 7 \%$ and a specificity of $80 \cdot 1 \%$. The positive predictive values of the tests listed in Table 3 were disa- ppointing but the negative predictive value was consistently very high. The positive and negative likelihood ratios were also consistently good, meaning that the tests are likely to modify pretest odds significantly and therefore give useful diagnostic information. ${ }^{25}$ For example, given no other information, the chances of a patient having a melanoma (the prevalence) in our sample was 1 in $7(15 \%)$ but, on performing SIAscopy and finding an absence of dermal melanin the odds drastically shifted to 83-1 against $(1 \cdot 2 \%)$. 
Table 2. Details of the dataset

\begin{tabular}{lc}
\hline Diagnosis & Number \\
\hline Melanoma & 52 \\
Superficial spreading & 41 \\
Nodular & 9 \\
Acral lentiginous & 2 \\
Common naevi (compound, & 185 \\
junctional and intradermal) & \\
Dysplastic naevi & 7 \\
Blue naevi & 12 \\
Spitz naevi & 7 \\
Seborrhoeic keratoses & 29 \\
Lentigo & 9 \\
Basal cell carcinoma & 21 \\
Dermatofibroma & 8 \\
Mixed naevi & 2 \\
Haemangioma & 2 \\
Others & 14 \\
Total & 348 \\
\hline
\end{tabular}

Using logistic regression analysis, receiver-operator characteristic (ROC) curves were constructed for combinations of features (Fig. 6). ROC curves are particularly useful when considering the performance of a diagnostic test, as they allow the user to assess its operating dynamics. By selecting a certain point on the ROC curve the clinician can see what the false-positive rate will be ( $x$-axis) at any given sensitivity ( $y$-axis). Thus, the ideal test describes a curve that goes from the origin to the top-left corner to the top-right corner, and the ambivalent (useless) test describes a diagonal line from the origin to the top-right corner (dotted line). In simple terms, the closer an ROC curve is to the top-left corner, the better the test can be considered to be. From the data it was possible to construct an ROC curve for the revised seven-point checklist; ${ }^{26}$ for comparison, the ROC curve for clinicians formally trained in dermatoscopy is also included. ${ }^{27}$ It can be seen from this graph that for different combinations of SIAscopy features the diagnostic performances are consistently much better than the revised seven-point checklist in detecting melanoma, and compare very well with dermatoscopy.

\section{Discussion}

This study assesses the clinical application of SIAscopy in the assessment of pigmented skin lesions. New diagnostic features that attempt to reveal the underlying histopathology have been identified by interpreting SIA graphs that return information about the concentration and position of collagen, blood and melanin within the epidermis and papillary dermis. Significant findings were the presence of blood displacement with erythematous blush, collagen holes and dermal melanin (Figs 2 and 3). An invasive nodule or nest of melanoma cells will displace blood from the papillary dermis, while early dermal invasion will promote a strong immunocytic response characterized by local vasodilatation and fibrosis. We hypothesize that this pathophysiological process is revealed by SIA graphs as erythematous blush with blood displacement. The papillary dermis is the reflective layer of the skin and any light that passes through this does not return. A melanoma that is Clark's level III or greater will, by definition, have areas where the papillary dermis is replaced by invasion. These areas can be seen in collagen SIA graphs as collagen holes (loosely termed as 'black holes' by our research group) where the

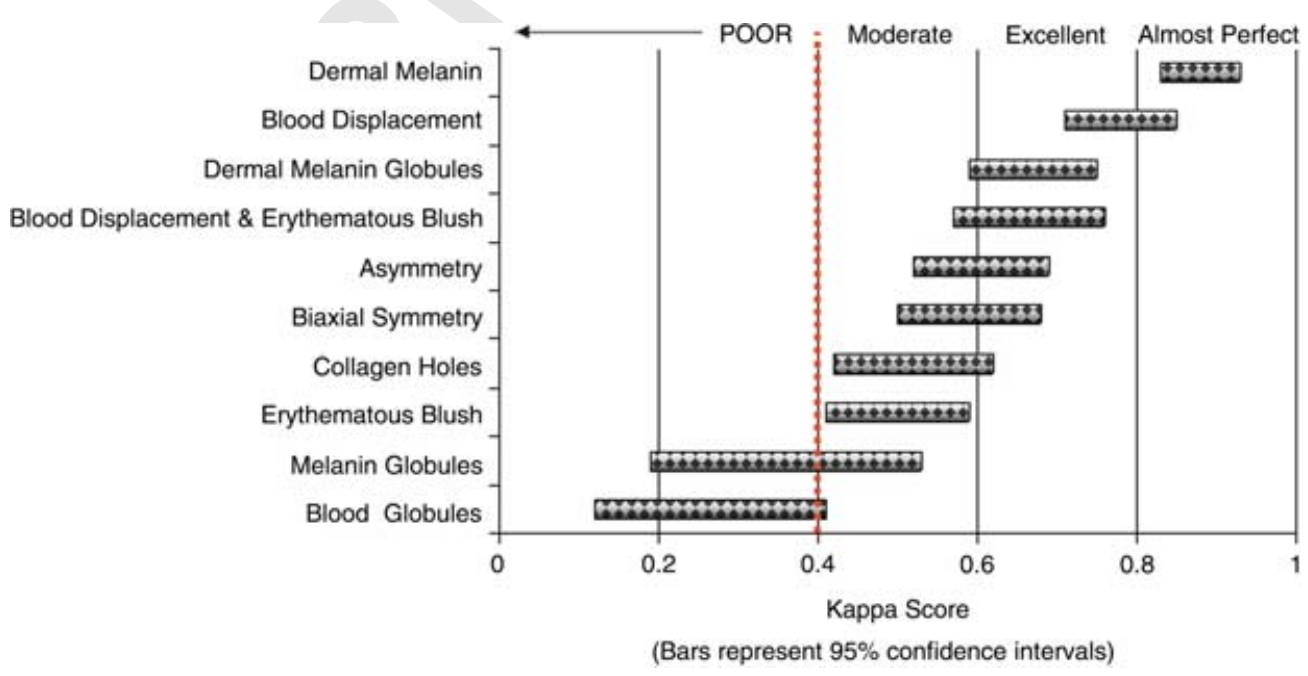

Figure 4. Reliability (interobserver agreement) of SIAscopy features. 


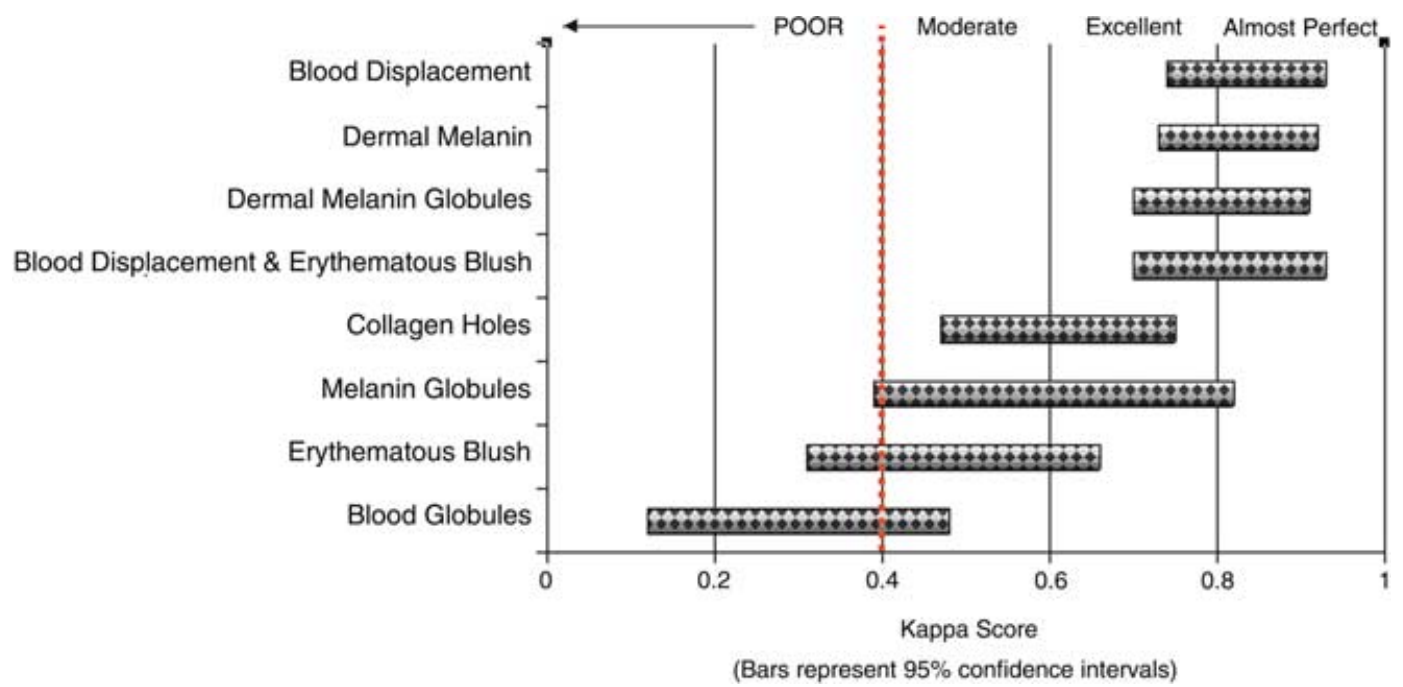

Figure 5. Reproducibility (intraobserver agreement) of SIAscopy features.

Table 3. Specificity and sensitivity analysis of SIAscopy features

\begin{tabular}{|c|c|c|c|c|c|c|}
\hline Feature & Sensitivity (\%) & Specificity (\%) & PPV (\%) & NPV (\%) & Positive LR & Negative LR \\
\hline \multicolumn{7}{|l|}{ Single features } \\
\hline Collagen holes & $78 \cdot 8(66-87 \cdot 8)$ & $74 \cdot 0(68 \cdot 7-78 \cdot 7)$ & $34 \cdot 7$ & $95 \cdot 2$ & $3.03(2 \cdot 39-3 \cdot 85)$ & $0 \cdot 29(0 \cdot 17-0 \cdot 49)$ \\
\hline Blood displacement & $75 \cdot 0(57 \cdot 3-87)$ & $70 \cdot 3(63-76 \cdot 6)$ & $30 \cdot 7$ & $94 \cdot 1$ & $2 \cdot 52(1.99-3 \cdot 19)$ & $0.36(0 \cdot 22-0.57)$ \\
\hline Erythematous blush & $75 \cdot 0(61 \cdot 8-84 \cdot 8)$ & $65 \cdot 5(60-70 \cdot 7)$ & $27 \cdot 7$ & $93 \cdot 7$ & $2 \cdot 18(1 \cdot 74-2 \cdot 72)$ & $0.38(0 \cdot 24-0 \cdot 62)$ \\
\hline $\begin{array}{l}\text { Blood displacement } \\
\text { with blush }\end{array}$ & $63 \cdot 5(49 \cdot 9-75 \cdot 2)$ & $84 \cdot 8(80 \cdot 3-88 \cdot 4)$ & $42 \cdot 3$ & 93 & $4 \cdot 17(2 \cdot 97-5 \cdot 86)$ & $0.43(0.3-0 \cdot 62)$ \\
\hline Dermal melanin & $96 \cdot 2(87-98 \cdot 9)$ & $56 \cdot 8(51 \cdot 1-62 \cdot 3)$ & $28 \cdot 1$ & $98 \cdot 8$ & $2 \cdot 22(1.93-2.56)$ & $0.07(0 \cdot 02-0 \cdot 26)$ \\
\hline Dermal melanin globules & $88 \cdot 5(77-94 \cdot 6)$ & $66 \cdot 6(61-71 \cdot 7)$ & $31 \cdot 7$ & 97 & $2 \cdot 64(2 \cdot 19-3 \cdot 19)$ & $0 \cdot 17(0 \cdot 08-0 \cdot 37)$ \\
\hline Asymmetry & $76 \cdot 9(63 \cdot 9-86 \cdot 3)$ & $62 \cdot 2(56 \cdot 5-67 \cdot 5)$ & $26 \cdot 3$ & $93 \cdot 9$ & $2 \cdot 03(1.65-2.5)$ & $0.37(0.22-0.61)$ \\
\hline Biaxial symmetry & $3 \cdot 8(1 \cdot 1-1 \cdot 3)$ & $70 \cdot 3(64 \cdot 8-75 \cdot 2)$ & $2 \cdot 2$ & $80 \cdot 6$ & $0.13(0.03-0.51)$ & $1.37(1.25-1.5)$ \\
\hline $\begin{array}{l}\text { Biaxial symmetry } \\
\text { not present }\end{array}$ & $96 \cdot 2(87-98 \cdot 9)$ & $29 \cdot 7(24 \cdot 8-35 \cdot 2)$ & $19 \cdot 4$ & $97 \cdot 8$ & $1 \cdot 37(1 \cdot 25-1 \cdot 5)$ & $0.13(0.03-0.51)$ \\
\hline \multicolumn{7}{|l|}{ Combined features } \\
\hline $\begin{array}{l}\text { Dermal melanin }+ \\
\text { collagen holes }\end{array}$ & $76 \cdot 9(63 \cdot 9-86 \cdot 3)$ & $78(73-82 \cdot 4)$ & $38 \cdot 1$ & $95 \cdot 1$ & $3 \cdot 5(2 \cdot 67-4 \cdot 52)$ & $0 \cdot 3(0 \cdot 18-0 \cdot 47)$ \\
\hline $\begin{array}{l}\text { Dermal melanin }+ \\
\text { displacement with blush }\end{array}$ & $96 \cdot 2(87-98 \cdot 9)$ & $56 \cdot 8(51 \cdot 1-62 \cdot 3)$ & $28 \cdot 1$ & $98 \cdot 8$ & $2.22(1.93-2.56)$ & $0.07(0 \cdot 02-0 \cdot 26)$ \\
\hline $\begin{array}{l}\text { Dermal melanin }+ \\
\text { displacement with } \\
\text { blush + biaxial } \\
\text { symmetry not present }\end{array}$ & $92 \cdot 3(81 \cdot 8-97)$ & $65 \cdot 9(60 \cdot 3-71)$ & $32 \cdot 2$ & 98 & $2 \cdot 71(2 \cdot 25-3 \cdot 23)$ & $0 \cdot 12(0 \cdot 05-0 \cdot 28)$ \\
\hline $\begin{array}{l}\text { Dermal melanin }+ \\
\text { displacement with blush }+ \\
\text { diameter } \geq 6 \mathrm{~mm}\end{array}$ & $82 \cdot 7(70 \cdot 3-90 \cdot 6)$ & $80 \cdot 1(75 \cdot 1-84 \cdot 2)$ & $42 \cdot 2$ & $96 \cdot 3$ & $2 \cdot 75(2 \cdot 13-3 \cdot 52)$ & $0 \cdot 25(0 \cdot 13-0 \cdot 43)$ \\
\hline
\end{tabular}

Values are given as mean (range). Sensitivity $=$ true positive $/($ true positive + false negative); specificity $=$ true negative $/($ true negative + false positive); positive predictive value $(\mathrm{PPV})=$ true positive/(true positive + false positive); negative predictive value $(\mathrm{NPV})=$ true negative $/($ true negative + false negative); positive likelihood ratio $(\mathrm{LR})=$ sensitivity percentage $/(100-$ specificity percentage $)$; negative LR $=(100-$ sensitivity percentage)/specificity percentage.

melanoma nests absorb or transmit light in the papillary dermis. The presence of dermal melanin in the papillary dermis, as expected, is a useful diagnostic sign, with a sensitivity of $96 \cdot 2 \%$, but the specificity was low at $56 \cdot 8 \%$. In compound and intradermal naevi, the dermal melanocytes begin to atrophy or 'mature' and cease to produce melanin ${ }^{5}$ and this fact will help in distinguishing benign naevi from melanoma. However, pigmentary incontinence and melanophages are found in both and are confounding factors adding to the high 


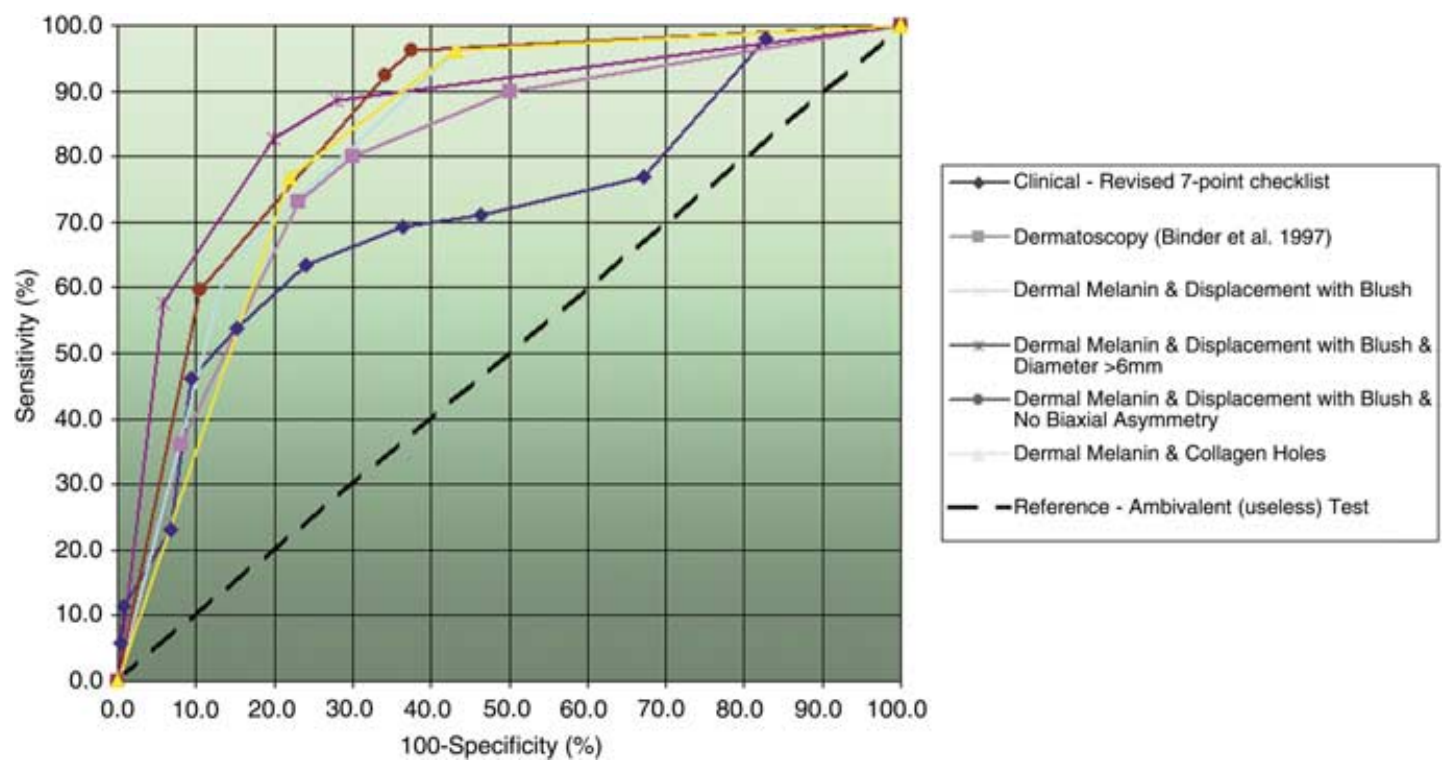

Figure 6. Receiver-operator characteristic (ROC) curves for combinations of SIAscopy features compared with clinical assessment and dermatoscopy (see text for explanation; dermatoscopy curve reproduced from Binder et al. ${ }^{27}$ with permission from the publishers, Harcourt Health Sciences).

false-positive rate as they will be observed in both sets of patients showing the presence of dermal melanin.

The results of this study indicate that the addition of in vivo information pertaining to the microscopic structure and architecture of a skin lesion can provide useful information in the diagnosis of melanoma. The presence or absence of features in the SIA graphs of pigmented skin lesions and the specificity and sensitivity data described compare very favourably with clinical examination and dermatoscopy. It is particularly worthwhile emphasizing that these early features described for SIAscopy of pigmented skin lesions are very simple to learn and use and this is reflected in the

1 reliability and reproducibility of $\kappa$ statistics. For instance, the mental process of detecting dermal melanin runs along the lines of 'is there any blue on the screen where the lesion is?' Similarly, the process of detecting blood displacement runs along the lines of 'is there white on the screen where the lesion is?' In contrast, due to the difficulty in interpreting subtle features, dermatoscopy requires formal training and experience before diagnostic accuracy improves. Furthermore, formally trained clinicians, experts and pioneers in the field often disagree on dermatoscopic findings in lesions, indicating a high subjectivity in interpreting features seen, most notably the highly significant finding of a blue/grey veil. ${ }^{28}$ Our specificity and sensitivity data of simple SIAscopic criteria compare very favourably against those published on clinicians after formal training in dermatoscopy ${ }^{27}$ in the diagnosis of cutaneous melanoma, especially when one analyses the ROC curves for the two techniques. In addition, combinations of features can greatly modify the pretest odds for melanoma and this attribute is particularly desirable in any diagnostic test. ${ }^{25} \mathrm{We}$ surmise that such an increase is due to the ability to identify specific processes occurring within a skin lesion confluent with melanoma such as the displacement of collagen by tumour cells and the vasodilatation and possibly angioneogenesis of papillary blood vessels.

Future work will produce probability data for diagnostic groupings or classes with certain combinations of features to facilitate the decision process for the clinician. The definitions used were deliberately designed as Boolean operators (i.e. 'true/false' or 'yes/ no') and these could be refined and stratified. As a result of the Boolean nature of the features, examination of the data did not include a 'pattern analysis' type approach to the assessment of the pigmented lesions that is so useful when performing a dermatoscopic diagnosis. Pattern analysis allows the clinician to take into account the overall appearance of the lesion and so helps to increase the specificity (reduce the false-positive rate). ${ }^{18,26}$ For example, in our dataset, blue naevi tended to be classified as melanomas because they usually demonstrated dermal melanin, collagen holes and blood displacement. However, the uniformity and regularity of these features and their 
sharp demarcation with the surrounding normal skin readily identifies them as blue naevi. Furthermore, the analysis was necessarily sterile and artificial in that no clinical diagnosis or dermatoscopic appearance was taken into account. Theoretically, it is reasonable to assume that the inclusion of such information would further improve the performance of the clinician.

A limitation of this study is the lack of direct comparison of the SIAscopy data with the dermatoscopy images. To become proficient in dermatoscopy requires formal training of at least $9 \mathrm{~h}^{19}{ }^{19}$ None of the authors had been formally trained in dermatoscopy and any attempts to diagnose melanoma solely from these images could lead to a worse performance than diagnosing pigmented lesions on clinical grounds alone. ${ }^{19}$ It was felt that it would be difficult to appraise the dermatoscopy images without biasing the performance of the technique due to lack of experience. Similarly, dermatoscopy is not universally practised in the U.K. and this may be due in part to an absence of formal training courses, while those that are available in continental Europe are not undertaken in English.

With a much larger dataset and with improving experience of SIAscopy, newer features will be described and tighter definitions will be developed. Methods that employ automated imaging analysis techniques are being investigated in order to increase reliability and reproducibility in the identification of SIAscopy features such as asymmetry and collagen holes. Additional studies are planned that will directly compare the performance of dermatoscopy with SIAscopy. As further studies are required to extend the number of lesions examined, an international study involving the U.K., Germany, Denmark and Australia is under way.

\section{Acknowledgments}

This research is funded by EPSRC grant no. GR/M53035.

\section{References}

1 Jones WO, Harman CR, Ng AK, Shaw JH. Incidence of malignant melanoma in Auckland, New Zealand: highest rates in the world. World J Surg 1999; 23: 732-5.

2 MacLennan R, Green AC, McLeod GR, Martin NG. Increasing incidence of cutaneous melanoma in Queensland, Australia. J Natl Cancer Inst 1992; 84: 1427-32.

3 Hall P, Javaid M, de Takats P. Therapeutic trends in cutaneous melanoma. Hosp Med 1999; 60: 39-43.
4 Creagan E. Malignant melanoma: an emerging and preventable medical catastrophe. Mayo Clin Proc 1997; 72: 570-4.

5 Mooi W, Krausz J. Biopsy Pathology of Melanocytic Disorders. London: Chapman \& Hall, 1992.

6 Elder D, Belle P, Elenitsas R et al. Neoplastic progression and prognosis in melanoma. Semin Cutan Med Surg 1996; 15: 336-48.

7 Curley R, Cook M, Fallowfield M, Marsden R. Accuracy in clinically evaluating pigmented lesions. Br Med J 1989; 299: 16-18.

8 Grin C, Kopf A, Welkovich B et al. Accuracy in the clinical diagnosis of malignant melanoma. Arch Dermatol 1990; 126: 763-6.

9 Morton C, MacKie R. Clinical accuracy of the diagnosis of cutaneous malignant melanoma. Br J Dermatol 1998; 138: 283-7.

10 El-Gammal S, El-Gammal C, Kaspar K et al. Sonography of the skin at $100 \mathrm{MHz}$ enables in vivo visualization of the stratum corneum and viable epidermis in palmar skin and psoriatic plaques. J Invest Dermatol 1999; 113: 821-9.

11 Pagnoni A, Kneuttel A, Welker P et al. Optical coherence tomography in dermatology. Skin Res Technol 1999; 5: 83-7.

12 Rajadhyaska M, Gonzalez S, Zavislan J et al. In vivo confocal scanning laser microscopy of human skin II. Advances in instrumentation and comparison with histology. J Invest Dermatol 1999; 113: 293-301.

13 Song $\mathrm{H}$, Wehrli $\mathrm{F}$, Ma J. In vivo MRI microscopy of human skin. Magn Reson Med 1997; 37: 185-91.

14 Bahmer F, Fritsch P, Kreusch J et al. Terminology in surface microscopy. J Am Acad Dermatol 1990; 23: 1159-61.

15 Mayer J. Systematic review of the diagnostic accuracy of dermatoscopy in detecting malignant melanoma. Med J Aust 1997; 167: 206-10.

16 Nachbar F, Stolz W, Merckle T et al. The ABCD rule of dermatoscopy. High prospective value in diagnosis of melanocytic lesions. J Am Acad Dermatol 1994; 30: 551-9.

17 Cristofolini N, Zumiani G, Bauer P et al. Dermatoscopy: usefulness in the differential diagnosis of cutaneous pigmentary lesions. Melanoma Res 1994; 4: 391-4.

18 Steiner A, Perhamberger $\mathrm{H}$, Wolff $\mathrm{K}$. In vivo epiluminescence microscopy of pigmented skin lesions and early detection of malignant melanoma. J Am Acad Dermatol 1987; 17: 584-91.

19 Binder N, Schwarz N, Winkler A et al. Epiluminescence microscopy. A useful tool for the diagnosis of pigmented lesions for formally trained dermatologists. Arch Dermatol 1995; 131: 286-91.

20 Cotton S. A Non-invasive Imaging System for Assisting in the Diagnosis of Malignant Melanoma. PhD Thesis. Faculty of Science, University of Birmingham, U.K. 1998.

21 Cotton SD, Claridge E, Hall PN. Noninvasive Skin Imaging

2 Information Processing in Medical Imaging. Springer-Verlag, 1997: 501-7.

322 Cotton SD, Claridge E. Developing a predictive model of human skin colouring. In: Proceedings of the SPIE Medical Imaging 1996 (Vanmetter RL, Beutel J, eds). Vol. 2708; 814-25.

23 Menzies S, Crotty K, Ingvar C, McCarthy W. An Atlas of Surface Microscopy of Pigmented Skin Lesions. Australia: McGraw-Hill, 1996.

24 Kianifard F. Evaluation of clinimetric scales: basic principles and methods. Statistician 1994; 43: 475-82.

25 Sackett D, Richardson W, Rosenberg W, Haynes B. Evidence Based Medicine-How to Practise and Teach EBM, 8th printing. Edinburgh: Churchill Livingstone, 1998: 118-28.

26 MacKie R. Clinical recognition of early invasive melanoma. Br Med J 1990; 301: 1005-6. 
27 Binder M, Puespoeck-Schwarz M, Steiner A et al. Epiluminescence microscopy of small pigmented skin lesions: short-term formal training improves the diagnostic performance of dermatologists. J Am Acad Dermatol 1997; 36: 197-202.
28 Soyer P, Argenziano G, Chimenti S et al. Dermoscopy of Pigmented Skin Lesions-An Atlas Based on the Consensus Net Meeting on Dermoscopy 2000. Milan: EDRA Medical Publishing, 2001. 


\section{Author Query Form}

\section{Journal: $\quad$ BJD}

Article: $\quad 4569$

Dear Author,

During the preparation of your manuscript for publication, the questions listed below have arisen. The numbers pertain to the numbers in the margin of the proof. Please attend to these matters and return the form with this proof.

Many thanks for your assistance.

\begin{tabular}{|l|l|l|}
\hline $\begin{array}{l}\text { Query } \\
\text { ference }\end{array}$ & re- & $\begin{array}{l}\text { Query } \\
\text { rks }\end{array}$ \\
\hline 1 & $\begin{array}{l}\text { Au: Is the text OK: this is reflected in the reliability and } \\
\text { reproducibility of ? statistics }\end{array}$ & \\
\hline 2 & Au: Please provide details of the publisher and town. & \\
\hline 3 & Au: 22 Cotton SD, Claridge E-year of publication? & \\
\hline 4 & Au: Australia-where in Australia? & \\
\hline
\end{tabular}




\section{Author Query Form}

\section{Journal: British Journal of Dermatology}

\section{Article: 4569}

Dear Author,

During the preparation of your manuscript for publication, the questions listed below have arisen. Please attend to these matters and return this form with your proof.

Many thanks for your assistance.

\begin{tabular}{|l|l|l|}
\hline Query Refs. & \multicolumn{1}{|c|}{ Query } & Remarks \\
\hline 1 & $\begin{array}{l}\text { Au: Is the text OK: this is reflected in the reliability } \\
\text { and reproducibility of ? statistics }\end{array}$ & \\
\hline 2 & $\begin{array}{l}\text { Au: Please provide details of the publisher and } \\
\text { town. }\end{array}$ \\
\hline 3 & $\begin{array}{l}\text { Au: } 22 \quad \text { Cotton SD, Claridge E-year of } \\
\text { publication? }\end{array}$ & \\
\hline 4 & Au: Australia—where in Australia? & \\
\hline
\end{tabular}

